\title{
Estudo das propriedades psicométricas do Addiction Severity Index (ASI): revisão sistemática da literatura*
}

Lídia Reis Fernandes ${ }^{1}$

(D) https://orcid.org/0000-0003-2837-6109

Laisa Marcorela Andreoli Sartes ${ }^{1}$

(D) https://orcid.org/0000-0002-1335-4305

Maira Leon Ferreira ${ }^{1}$

(DD https://orcid.org/0000-0002-7400-304X

\footnotetext{
* Artigo extraído da tese de doutorado "Validação da versão brasileira do Addiction Severity Index 6 (ASI6) Light", apresentada ao Instituto de Ciências Humanas, Universidade Federal de Juiz de Fora, Juiz de Fora, MG, Brasil.

1 Universidade Federal de Juiz de Fora, Instituto de Ciências Humanas, Juiz de Fora, MG, Brasil.
}

Objetivo: realizar uma revisão sistemática da literatura das propriedades psicométricas do ASI. Método: foram incluídos estudos indexados nas bases de dados Pubmed, PsycInfo, Scielo e Lilacs, que abordavam estudos de avaliação psicométrica da quinta e sexta versão do ASI. Resultados: descritos em categorias classificadas quanto ao número de métodos de análises, mostram manuscritos de diversos países e em diferentes contextos que utilizaram métodos clássicos para as análises de validação e confiabilidade da quinta e da sexta versão do ASI. De uma maneira geral, ambas as versões apresentaram boas propriedades psicométricas, embora algumas áreas, como por exemplo, a área "familiar", apresentaram algumas limitações quanto à confiabilidade e a consistência interna. A maioria dos estudos mostra uma correlação moderada entre os escores sumários do ASI e outros instrumentos, não sendo uniformemente alta. Entretanto as áreas "álcool" e "drogas" foram as que mais apresentaram altas correlações com outros instrumentos considerados medidas externas. As análises apoiaram a multidimensionalidade do ASI, porém, tiveram algumas exceções. Conclusão: apesar das limitações, o ASI é utilizado amplamente em todo o mundo, por profissionais de diferentes áreas, psicólogos, enfermeiros, assistentes sociais e psiquiatras. Tem auxiliado clínicos e pesquisadores, contribuindo para a elaboração de um plano terapêutico, determinando as prioridades clínicas, bem como o manejo dessas.

Descritores: Validação; Confiabilidade; Escalas; Substâncias Psicotrópicas.

\section{Como citar este artigo}

Fernandes LR, Sartes LMA, Ferreira ML. Estudo das propriedades psicométricas do Addiction Severity Index (ASI): revisão sistemática da literatura. SMAD, Rev Eletrônica Saúde Mental Álcool Drog. 2020;16(2):1-11. doi: https://dx.doi.org/10.11606/issn.1806-6976.smad.2020.159845 


\section{Study of psychometric properties of do Addiction Severity Index (ASI): systematic literature review}

Aim: to perform a systematic literature review of psychometric properties of the ASI. Method: were included studies indexed in the databases Pubmed, PsycInfo, Scielo and Lilacs, that approached studies of psychometric evaluation of quinine and sixth version of ASI. Results: described in categories classified according to the number of methods of analysis, show manuscripts from different countries and in different contexts that used classic methods for the validation and reliability analyzes of the fifth and sixth version of ASI. Overall, both versions had good psychometric properties, although some areas, such as the "family" area, presented some limitations on reliability and internal consistency. Most studies show a moderate correlation between ASI summary scores and other instruments, not being uniformly high. However, the areas "alcohol" and "drugs" were the ones that presented the highest correlations with other instruments considered external measures. The analyzes supported the multidimensionality of ASI, however, with some exceptions. Conclusion: despite the limitations, ASI is used widely throughout the world by professionals from different fields, psychologists, nurses, social workers and psychiatrists. It has helped clinicians and researchers, contributing to the elaboration of a therapeutic plan, determining the clinical priorities, as well as the management of these.

Descriptors: Validation; Reliability; Scales; Psychotropic Substances.

\section{Estudio de las propiedades psicométricas del do Addiction Severity Index (ASI): revisión sistemática de la literatura}

Objetivo: realizar una revisión sistemática de la literatura sobre las propiedades psicométricas de ASI. Método:se incluyeron los estudios indexados en las bases de datos Pubmed, PsycInfo, Scielo y Lilacs, que abordaron los estudios de evaluación psicométrica de quinina y la sexta versión de ASI. Resultados: descritos en categorías clasificadas según el número de métodos de análisis, muestran manuscritos de diferentes países y en diferentes contextos que utilizaron métodos clásicos para los análisis de validación y confiabilidad de la quinta y sexta versión de ASI. En general, ambas versiones tenían buenas propiedades psicométricas, aunque algunas áreas, como el área "familiar", presentaban algunas limitaciones en cuanto a confiabilidad y consistencia interna. La mayoría de los estudios muestran una correlación moderada entre los puntajes de los resúmenes ASI y otros instrumentos, que no son uniformemente altos. Sin embargo, las áreas "alcohol" y "drogas" fueron las que presentaron las mayores correlaciones con otros instrumentos considerados medidas externas. Los análisis apoyaron la multidimensionalidad de ASI, sin embargo, con algunas excepciones. Conclusión: a pesar de las limitaciones, el ASI se usa ampliamente en todo el mundo por profesionales de diferentes campos, psicólogos, enfermeras, trabajadores sociales y psiquiatras. Ha ayudado a clínicos e investigadores, contribuyendo a la elaboración de un plan terapéutico, determinando las prioridades clínicas y el manejo de estos.

Descriptores: Validación; Fiabilidad; Escalas; Sustancias Psicotrópicas. 


\section{Introdução}

O Addiction Severity Index (ASI) é um dos instrumentos de avaliação mais utilizado no campo do abuso de substância(1). Foi desenvolvido em 1980 por McLellan et al. A escala avalia sete áreas da vida de pessoas com problemas associados ao abuso e dependência de álcool e drogas: problemas médicos, situação ocupacional, aspectos legais, sociofamiliares, psiquiátricos, uso de álcool e uso de outras drogas.

O ASI foi desenvolvido inicialmente para propósitos de pesquisa. A partir de então, várias versões do instrumento foram desenvolvidas, sendo amplamente aceito e utilizado em diversas partes do mundo(2-7). É uma ferramenta apropriada principalmente para as seguintes finalidades: determinar áreas problemáticas, fornecer uma entrevista técnica estruturada e avaliar a necessidade subjetiva de tratamento em vários domínios.

A quinta versão do ASI é uma entrevista semiestruturada, que deve ser realizada por entrevistadores treinados e em que o tempo de aplicação é relativamente breve (entre 45 a 90 minutos) considerando a amplitude de informações que fornece. É dividida nas sete áreas acima citadas, cada área é formada por questões objetivas sobre o número, a intensidade e a duração dos sintomas ocorridos durante toda a vida do indivíduo e nos últimos 30 dias que antecedem a entrevista. Há duas questões que são realizadas no final de cada área para que o paciente faça uma avaliação subjetiva do grau de preocupação com os problemas e sintomas atuais e a necessidade de tratamento para esses problemas. A partir dessas informações, o entrevistador define o Escore de Gravidade do Entrevistador (Interviewer Severity Ratings) variando de 0 a 10, que determina o nível de gravidade em cada área e a necessidade de tratamento adicional. O Escore Composto (Composite Score) é obtido a partir dos dados objetivos, incluindo apenas os itens passiveis de mudanças: problemas ocorridos nos últimos 30 dias ou no período de seguimento( ${ }^{(8)}$.

Visando avaliar as propriedades psicométricas do ASI até a quinta versão, foi realizada uma revisão de 37 estudos, de maneira bastante crítica, em que foi encontrada uma grande variação na confiabilidade dos Escores de Gravidade e Escores Compostos entre entrevistadores e após teste-reteste ${ }^{(9)}$. Tal diferença foi atribuída, em grande parte, ao uso em diferentes populações e países, assim como aos diferentes níveis de treinamento dos entrevistadores.

A sexta versão do instrumento foi desenvolvida no intuito de minimizar as limitações presentes nas versões anteriores do ASI, atualizar e expandir suas áreas de avaliação. Além disso, nas últimas décadas, houve profundas mudanças na natureza das substâncias psicoativas disponíveis, bem como nas características do paciente e do tratamento(10). O ASI6 é composto por 252 questões, também agrupadas nas mesmas sete áreas de avaliação. Foram acrescentados à sexta versão, alguns itens relacionados ao uso do tempo livre e histórico de traumas. Os períodos de tempo de vida e últimos 30 dias continuam sendo os principais intervalos de avaliação. No entanto, uma crítica tem sido que o prazo de 30 dias é muito breve para avaliar adequadamente a base funcional e muito impreciso para avaliar a acuidade do problema recente. Para resolver essas limitações, o ASI6 inclui um período de seis meses e uma data do último teste de ocorrência para itens-chave ${ }^{(10)}$. Além disso, foi retirada a avaliação subjetiva de problemas feita pelo entrevistador, que era ponderada com a do paciente nas versões anteriores, tornando os Escores de Gravidade do Entrevistador baseados apenas na avaliação do paciente, denominado agora de Escores de Gravidade do Entrevistado. Os Escores Compostos (ECs) foram substituídos pelos Summary Scores for Recent Functioning (SS-Rs - Escores Resumo de Funcionamento Recente, em português), que tem por base as questões sobre problemas ocorridos nos últimos 30 dias.

Nesse contexto, o objetivo deste trabalho foi realizar uma revisão da literatura de maneira sistemática dos estudos que avaliam as propriedades psicométricas do ASI a fim de obter um panorama geral dos métodos de análises utilizados nos últimos anos desde a última revisão realizada em 2004(9).

\section{Método}

A revisão foi conduzida de acordo com os passos indicados pelo e Preferred Reporting Items for Systematic Reviews and Meta-Analyses (PRISMA)(11). As buscas foram realizadas em julho de 2018. Com o objetivo de avaliar as experiências mais recentes, incluímos nas buscas estudos completos e publicados no período de 2005 a 2018. A justificativa da escolha das bases de dados se deu pela relação do tema com o conteúdo indexado. Para melhor definição dos termos de busca nas bases selecionadas, foram utilizadas palavras-chave indexadas pelas próprias bases através de procedimentos de controle de vocabulário. Portanto, os termos da pesquisa variaram conforme a base de dados a fim de garantir uma busca mais efetiva dos estudos. Para cobrir o maior número de estudos possível, conduzimos buscas nas seguintes bases de dados: Pubmed, PsycInfo, Scielo e Lilacs. Os idiomas pesquisados foram de acordo com o idioma principal de cada base de dados, abrangendo as línguas inglesa, portuguesa e espanhola.

Para a pesquisa na Pubmed e Psycinfo os termos utilizados foram na língua inglesa considerando a língua original da base de dados: "addiction severity index and validity", "addiction severity index and reliability", "addiction severity index and sensitivity" e "addiction severity index and specificity". Estes itens também 
foram incluídos na pesquisa para as bases Scielo e Lilacs. Para a base Scielo, além dos termos em inglês citados acima, foram utilizados os seguintes descritores em português: "escala de gravidade de dependência e validação", "escala de gravidade de dependência e confiabilidade", "escala de gravidade de dependência e sensibilidade" e "escala de gravidade de dependência e especificidade". Para a Lilacs, além dos termos em inglês citados anteriormente, os termos utilizados em espanhol foram: "addiction severity index y validación", "addiction severity index y confiabilidad", "addiction severity index y sensibilidad", "addiction severity index y especificidad".

Foram critérios de inclusão: artigos empíricos que estivessem nas línguas inglesa, espanhola e portuguesa que abordasse no título ou no resumo os termos ASI ou "addiction severity index" e estudos de validação. Para este estudo as versões do ASI 5 e 6 foram consideradas para análise, bem como as adaptações dessas versões para outros países. Foram incluídos no estudo pesquisas que envolviam todos os tipos de drogas, ou cada droga de forma isolada.

Foram excluídos deste estudo as teses e dissertações, livros e capítulo de livros, como também, trabalhos publicados nos idiomas que não eram o inglês, o espanhol e o português e trabalhos que não possuíam o artigo completo disponível.

Após exclusão dos artigos duplicados e dos manuscritos que não se adequaram aos critérios de inclusão estabelecidos acima, estabeleceu-se o total de manuscritos que foram incluídos para a análise final. Estas publicações foram, inicialmente, classificadas pelos seguintes indicadores bibliométricos: autoria, ano de publicação, idioma de publicação, número de participantes, principal droga, instrumentos e métodos utilizados.

\section{Resultados}

A busca inicial nas bases de dados recuperou 500 artigos que foram exportados para o software livre My Endnote Web. Foram excluídos 172 artigos, a partir da ferramenta do próprio Endnote para encontrar duplicações. Os 328 artigos restantes foram submetidos à leitura, por pares, dos títulos e resumos. Destes, foram excluídos 293 manuscritos categorizados em: não correspondem ao objetivo do presente estudo, artigos sobre efetividade e eficácia de programas psicoterapêuticos, artigos sobre propriedades psicométricas de outros instrumentos e artigos escritos em outros idiomas.

Uma segunda etapa envolveu a leitura integral dos 35 estudos restantes, a fim de averiguar se, de fato, eles cumpriam os critérios de inclusão. Ao todo, 19 estudos não puderam ser incluídos, sendo que destes, 10 artigos não avaliaram as propriedades psicométricas do ASI e nove manuscritos não possuíam texto completo disponível. Após esta etapa de exclusão dos artigos, restaram 16 publicações que cumpriram todos os critérios de inclusão (Tabela 1). Os indicadores bibliométricos foram tabulados no Microsoft Excel.

A Figura 1 apresenta o fluxograma (PRISMA flow diagram) sobre as buscas e análises realizadas.

Tabela 1 - Artigos incluídos para análise dos estudos das propriedades psicométricas do ASI. Juiz de Fora, Minas Gerais, Brasil, 2018

\begin{tabular}{|c|c|c|c|c|c|}
\hline Artigos & País & Amostra N & Principal droga & Instrumentos & Métodos* \\
\hline Gerevich J, et al., (2005) & Hungria & 266 & Drogas em geral & ASI5; EuropASI; BDI & CEA, CTR, CI, IA, EV \\
\hline Reelick N et al., (2006) & $\begin{array}{l}\text { Países } \\
\text { Baixos }\end{array}$ & 1027 & Heroína & ASI5 (área psiquiátrica) & IC \\
\hline Rikoon S et al., (2006) & EUA & 2824 & Drogas em geral & ASI5; DSM IV & IC \\
\hline Alterman et al., (2007) & EUA & 2142 & Drogas em geral & ASI5 & IA, EV \\
\hline Cacciola J et al., (2007) & EUA & 195 & Drogas em geral & ASI5; ASI L-VA & CTR, CI, IA, EV \\
\hline Haraguchi A, et al., (2009) & Japão & 370 & Álcool & $\begin{array}{l}\text { ASI-J versão do ASI5; Escala de atitudes } \\
\text { indesejáveis ao tratamento }\end{array}$ & $\mathrm{CI}, \mathrm{IA}, \mathrm{IC}, \mathrm{EV}$ \\
\hline Luo W, et al., (2010) & China & 526 & Drogas em geral & $\begin{array}{l}\text { ASI5, SCL-90, } \\
\text { FACES } \alpha-C V\end{array}$ & CEA, CTR, CI, IA, EV \\
\hline Días Mesa E et al., (2010) & Espanha & 253 & Drogas em geral & ASI6; ICG-G & CTR, Cl, EV \\
\hline Cacciola et al., (2011) & EUA & 254 & Drogas em geral & ADI6 & $I A, E V, E C$ \\
\hline Kessler F, et al., (2012) & Brasil & 740 & Drogas em geral & ASI6, WHOQOL, ASSIST, SAS-SR & CEA, CTR, CI, IA, EV \\
\hline Sun, $Z$ et al., (2012) & China & 254 & Álcool & ASI-C-5; AUDIT & CTR, CI, IA, EV, IC \\
\hline Wei L, et al., (2012) & China & 380 & Drogas em geral & $\begin{array}{c}\text { ASIC (baseado no ASI5); SCL-90; FACES } \\
\text { II - CV }\end{array}$ & CTR, CI, IA, EV \\
\hline López-Goñi J et al., (2012) & Espanha & 252 & Drogas em geral & EuropASI & IC \\
\hline Denis M, et al., (2013) & EUA & 607 & Drogas em geral & ASI5; ASI6 & $\mathrm{IA}, \mathrm{EV}$ \\
\hline Demirbas H, et al., (2014) & Turquia & 115 & Álcool & ASI5, BDI, MAC & CTR, Cl, EV \\
\hline Fernandes L, et al., (2015) & Brasil & 200 & Drogas em geral & ASI6; ASSIST & IC, EV \\
\hline
\end{tabular}

*CEA = Confiabilidade entre avaliadores; CTR = Confiabilidade teste-reteste; CI = Consistência interna; IA = Independência das áreas; EV = Evidências de validade; IC = Índices clínicos; EC = Escore composto 


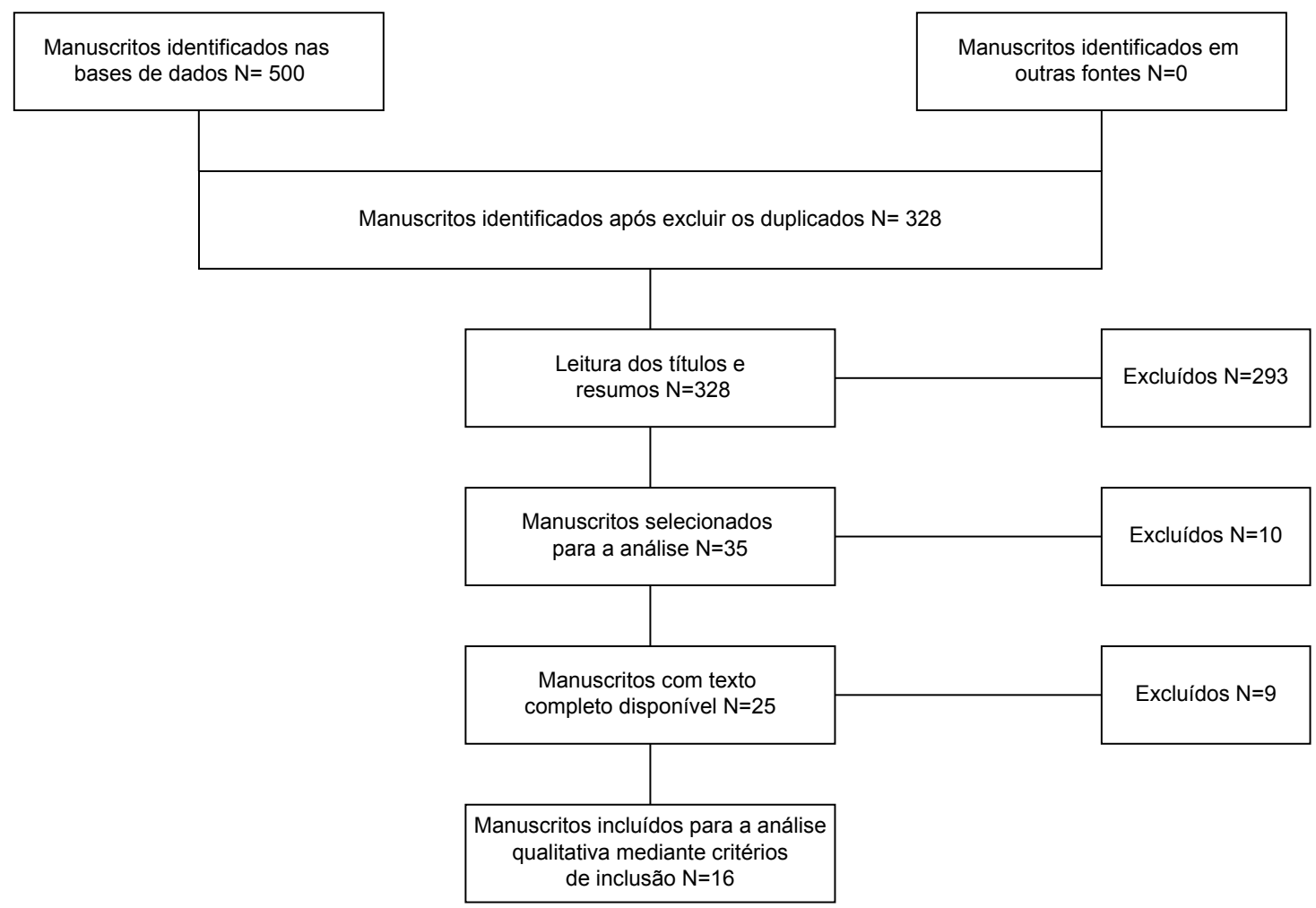

Figura 1 - Fluxograma de identificação e seleção dos artigos para as análises

As categorias foram classificadas em número de métodos de análises conforme descritas a seguir:

\section{Quatro ou mais métodos de análises}

Nesta categoria foram incluídos sete artigos que correspondem ao período de 2005 a 2014. O principal grupo avaliado utilizava vários tipos de drogas, que emergiu em quatro estudos, e o álcool, em dois trabalhos. Os estudos buscavam, de forma geral, investigar as propriedades psicométricas do ASI, adaptar esse instrumento para outras línguas, bem como testar sua validade e confiabilidade. Todos os estudos foram publicados na língua inglesa, porém, dois estudos foram realizados na China, um no Japão, um nos Estados Unidos, um na Hungria, um na Turquia e outro no Brasil. As pesquisas foram predominantemente transversais, 0 tamanho da amostra variou de 195 a 740 participantes. A versão do ASI5 foi utilizada em seis dos sete estudos analisados, um deles utilizou o ASI6.

No geral, altos índices de confiabilidade entre os entrevistadores foram obtidos para os escores de gravidade e os escores compostos variando de 0,74 a $0,98^{(5,7)}$. Portanto, os escores compostos apresentaram coeficientes de Pearson maiores do que os escores de gravidade do entrevistador.

Em um estudo realizado para a avaliação psicométrica da versão Húngara do EuropASI, para avaliar a confiabilidade teste-reteste, a entrevista com o ASI foi repetida (3-8 dias) após a primeira entrevista em 25 pacientes. As correlações gerais para os escores compostos tiveram uma confiabilidade teste-reteste variando de 0,37 a 0,92. As áreas "família" $(r=0,37)$ e "médica" $(r=0,44)$ não demonstraram boa confiabilidade teste-reteste nesta amostra de entrevistados( ${ }^{(5)}$.

Ao avaliar as características psicométricas da tradução turca do ASI5 em 115 pacientes dependentes de álcool do sexo masculino, os pesquisadores realizaram uma análise de confiabilidade teste-reteste com um intervalo de 10 dias com 30 indivíduos selecionados aleatoriamente. Foram encontradas as seguintes correlações de Spearman $\rho: 0,85$ para a área "médica", 0,79 para a área "emprego", 0,84 para a área "legal", 0,85 para área "álcool", 0,83 para área "sócio-familiar" e 0,91 para área "psiquiátrica"(12).

Para avaliar as propriedades psicométricas da versão brasileira da sexta versão do ASI, foi realizado um estudo transversal e multicêntrico(13). Uma subamostra aleatória de 51 pacientes foi solicitada a repetir a entrevista inicial usando o ASI6 com um entrevistador diferente em um período que variou de três a sete dias após a primeira entrevista, a fim de verificar a confiabilidade teste-reteste do instrumento. As medidas não mostraram diferenças entre os índices sumários de ambas as entrevistas, exceto para a área "emprego" $(\mathrm{CCI}=0,73)$. As diferenças entre as médias dos grupos também foram calculadas de acordo com o tratamento e não houve significância estatística no grupo de internação, enquanto diferenças significativas foram encontradas no grupo ambulatorial em relação à área "emprego" ( $p=0,008$ e tamanho do efeito $=0,61)$ e "apoio familiar/social" (0,038 e tamanho do efeito $=0,43$ ). 
Em um estudo conduzido para realizar a validação da versão chinesa do ASI5, obteve-se uma amostra de 354 pacientes internados dependentes de álcool. Entre esses, quarenta foram selecionados aleatoriamente para participarem de medidas teste-reteste, em um intervalo de sete dias, com o mesmo entrevistador. A correlação entre os coeficientes de Pearson para escores compostos variou de 0,75 a 0,92, indicando um nível moderado a alto de estabilidade dos escores ASI-C entre pacientes com histórico de dependência de álcool(14).

Em um estudo realizado para testar a confiabilidade e a validade da versão chinesa do ASI5 em uma amostra de pacientes com MMT (tratamento de manutenção com metadona), para as medidas teste-reteste foi utilizado uma amostra de 51 pacientes. Os escores compostos (ECs) antes e depois do intervalo de uma semana foram altamente correlacionados. As correlações de Spearman para o teste-reteste foram de moderadas a altas $(0,68-0,84)$. Nas áreas "psiquiátrica" $(\rho=0,68)$, "drogas" $(\rho=0,70)$ e "legal" $(\rho=0,72)$ as correlações foram moderadas ${ }^{(7)}$.

A consistência interna para a área "emprego" e área "álcool" apresentou valores problemáticos em três estudos dessa categoria(7,14-15). Os coeficientes variaram de 0,48 a 0,67 para a área "emprego" e de 0,67 a 0,68 para a área "álcool". Foram dois estudos chineses e um japonês.

O estudo de validação da versão brasileira do ASI6 demonstrou consistências internas relativamente altas para oito dos nove índices sumários (drogas, família/ filhos, álcool, psiquiátrica, médica, legal, emprego e família/suporte social) variando de 0,64 a 0,95. A área "família/problema social" apresentou consistência interna baixa $(a=0,64)^{(13)}$.

Em um estudo que tinha como objetivo avaliar as propriedades psicométricas de uma versão abreviada do ASI5, o ASI-L-VA, as correlações entre as áreas problemáticas foram geralmente baixas para ambas as versões ${ }^{(16)}$. Nenhum dos testes t comparando as correlações correspondentes entre o ASI5 e o ASI-L-VA aproximou-se da significância estatística (todos p's > 0.20).

No estudo realizado para validar a versão chinesa do ASI5(7), com uma amostra de pacientes em tratamento de manutenção com metadona, a correlação do escore psiquiátrico com a Escala de Avaliação de Sintomas 90-R (SCL-90-R) foi de 0,50. A correlação do escore familiar/ social com a Escala de Avaliação da Adaptabilidade Familiar e Coesão a-versão chinesa (FACES a-CV) foi de 0,45.

O estudo da versão Húngara do EuropASI ${ }^{(5)}$, com uma amostra 266 pacientes usuários de álcool e drogas em tratamento, em cada área de problema, vários itens foram selecionados como indicadores de gravidade do problema e as intercorrelações entre esses itens e as classificações de severidade foram determinadas. As classificações de gravidade psiquiátrica foram moderadamente correlacionadas com a pontuação média do Inventário de Depressão de Beck e a subescala de Humor Negativo $(r=0,43)$.

A validade de critério da versão chinesa do ASI5 foi mensurada em uma amostra de 354 pacientes internados com dependência de álcool. Para avaliar a validade de critério, foram observados coeficientes de correlação de 0,4 a 0,8, considerados aceitáveis. A correlação entre os escores de uso de álcool medido pelo ASI-C-5 e Alcohol Use Disorders Identification Test (AUDIT) foi de 0,69 $(p<0,01)$, indicando validade de critério significante do ASI-C-5 no domínio do uso de álcool(14).

A correlação do escore psiquiátrico com o SCL-90-R foi de 0,56 ( $p<0,001)$ e a correlação do escore familiar/ social com o Family Adaptability and Cohesion Evaluation Scale (FACES II) foi de 0,26 ( $p<0,05)$. A validade de critério foi medida em uma amostra de 381 usuários de drogas na comunidade de Chengdu, na província de Sichuan, em um estudo cujo objetivo era avaliar a confiabilidade e validade de uma nova versão chinesa do Addiction Severity Index (ASI-C) em usuários de drogas na comunidade ${ }^{(7)}$.

Em um estudo em que o objetivo era testar as propriedades psicométricas da sexta versão do $\mathrm{ASI}^{(13)}$, correlações entre os escores da área álcool e drogas do ASI6 e o instrumento Alcohol Smoking and Substance Involvement Screening Test (ASSIST) foram altas $(0,72$ e 0,89 , respectivamente). Houve correlação negativa significativa entre os escores psiquiátrico e médico em relação àqueles do Word Health Organization Quality of Life (WHOQOL) $(-0,76$ e $-0,41$, respectivamente). Foi encontrada uma correlação positiva entre os escores da Social Adjustment Scale (SAS) e a área "família/suporte social" do ASI6 $(0,47)$.

\section{De dois a três métodos de análises}

Os estudos encontrados nessa categoria de análise totalizaram sete manuscritos. O período de publicação foi de 2006 a 2015. Seis manuscritos avaliaram usuários de drogas em geral, um deles utilizou usuários de heroína. Os estudos também possuíam como objetivo investigar as propriedades psicométricas do ASI, testar sua validade e confiabilidade e adaptar essa escala para outros países. Dos sete estudos publicados, cinco foram publicados na língua inglesa, um na língua espanhola e um deles na língua portuguesa. Destes estudos três foram realizados nos Estados Unidos, um nos Países Baixos, um na Espanha, um na China e outro no Brasil. Em relação ao delineamento, os sete estudos foram transversais, e um deles utilizou o método observacional e prospectivo. O tamanho da amostra variou de 115 a 2142 participantes. Dos sete artigos, três utilizaram a versão do ASI5, um deles utilizou o ASI5 e o ASI6, e três utilizaram o ASI6. 
Em um estudo realizado para avaliar as propriedades psicométricas de uma nova versão chinesa do Addiction Severity Index (ASI-C) em usuários de drogas na comunidade, 38 indivíduos foram entrevistados novamente, em um intervalo de sete dias, para avaliar a confiabilidade teste-reteste. As correlações de Spearman variaram de 0,50 para os escores da área "psiquiátrica" a 0,93 para a área "Legal"(17).

No estudo para avaliar as propriedades psicométricas do ASI6 em sua versão traduzida e adaptada para o espanhol, em termos de confiabilidade teste-reteste, o tempo médio entre as entrevistas foi de 8 a 21 dias. O coeficiente de correlação de Spearman $r$ entre os escores de teste e reteste variou de 1,00 (famíliacrianças) e 0,96 (apoio-relacionamento com parceiros) a 0,37 (problemas de relacionamento com adultos e 0,36 (problemas de relacionamento com amigos), e todas foram estatisticamente significativas, exceto o último ${ }^{(18)}$.

Em um estudo com o foco na área psiquiátrica do ASI5, teve como objetivo avaliar o poder de previsão do uso de cuidados de saúde mental por pacientes com problemas por uso de substâncias. Foram entrevistados 1052 sujeitos que fazem o uso de heroína. Os autores utilizaram três índices: o índice de avaliação, o índice clínico e os escores compostos. Chegaram à conclusão que a escala psiquiátrica discrimina entre aqueles que terão contato com a saúde mental e aqueles que não terão. No entanto, a porcentagem de falsos positivos é alta e nenhum dos índices prevê a intensidade e a duração do tratamento de saúde mental(19).

Com o objetivo de descrever a derivação de índices resumo de problemas recentes (Summary Scores for Recent Functioning/SS-Rs) do ASI6, foi utilizada uma amostra de 607 pacientes que faziam tratamento em programas de uso de substâncias para o estudo de derivação, e um subconjunto $(\mathrm{N}=254)$ compreendeu a amostra para um estudo de validação. Um total de 118 itens relacionados à avaliação de problemas recentes do ASI6 foi submetido à análise de teoria de resposta de item não paramétrica (NIRT) seguida de análise fatorial confirmatória (CFA). Foram derivados nove índice resumo confiáveis e dimensionalmente homogêneos; uma escala para cada uma das seis áreas (médica, emprego/finanças, álcool, drogas, legal, psiquiátrica) e três escalas para a família/área social. Além disso, análises de validade concorrente produziram fortes evidências apoiando a validade de seis SS-RS (médico, álcool, drogas, emprego, família/problemas sociais, psiquiátrica). A evidência foi mais fraca para os SS-Rs de apoio Legal, familiar/social e problemas de relacionamento com crianças $^{(10)}$.

Foi realizado um estudo com uma amostra de 2142 indivíduos que fazem uso abusivo de substâncias para gerar índices resumo de problemas recentes e de história de vida das sete áreas do ASI5. Foram utilizados dois métodos não-paramétricos de resposta ao item (NIRT): escala de Mokken e técnicas de covariância condicional. No total, 14 índices foram derivados, sendo nove relacionados a problemas recentes das sete áreas do ASI5 e cinco relacionados a problemas ao longo da vida, exceto das áreas "emprego/sustento" e "família/ social". O número de itens incluídos para formação destes índices variou entre as áreas. Por exemplo, três itens da área "médica" e sete da área "drogas" formaram os índices de problemas recentes, cujos valores foram respectivamente $\mathrm{H}=0,80$ e $\mathrm{H}=0,71$. Para a formação dos índices referentes a histórico de problemas de cada área, cinco itens foram incluídos da área "álcool" $(\mathrm{H}=0,67)$ e dez da "psiquiátrica" $(\mathrm{H}=0,49)^{(20)}$.

Um estudo foi realizado para comparar as características e a validade dos escores compostos (ECs) do ASI5 e os índices resumo de problemas recentes (SSRs) do ASI6 em uma amostra de pacientes de programas de tratamento de abuso de substância entrevistados tanto com o ASI5 quanto com o ASI6. Ambas as intercorrelações entre os nove SS-Rs do ASI6 e entre os sete ECs do ASI5 são, como esperado, geralmente baixas. No entanto, houve cinco correlações significativas entre os SS-Rs: Legal com drogas $(\rho=0,47)$; psiquiátrico com médico $(\rho=0,43)$ e drogas $(\rho=0,35)$; família/ problemas sociais com drogas $(\rho=0,42)$ e psiquiátrico $(\rho=0,34)$. Houve duas correlações significativas entre os ECs do ASI5, a área "psiquiátrica" foi correlacionada com as áreas "médica" $(\rho=0.35)$ e "drogas" $(\rho=0.31)$. Nenhum dos coeficientes de correlação entre SS-Rs e ECs foi significativamente diferente ${ }^{(21)}$.

Em um estudo brasileiro, que entrevistou 200 usuários de drogas, com o objetivo de avaliar a validade de critério das áreas álcool e drogas de uma versão brasileira reduzida do ASI6 (ASI6 Light) foi encontrada alta correlação entre os escores da área "álcool" do ASI6 Light e os escores do ASSIST em relação ao álcool $(r=0,79)$, correlações moderadas em relação ao tabaco $(r=0,47)$ e cocaína/crack $(r=0,44)$ e baixa $(r=0,39)$ em relação à maconha. Ao correlacionarem-se os escores do ASSIST e os escores da área "drogas" do ASI6 Light, obteve-se alta correlação em relação à cocaína/crack $(r=0,85)$, correlações moderadas em relação ao tabaco $(r=0,57)$ e maconha $(r=0,68)$ e baixa $(r=0,29)$ em relação ao álcool(22).

\section{Um método de análise}

Nesta categoria foram adicionados dois estudos que utilizaram somente um método de análise. Um dos estudos foi correlacional, publicado em 2006 nos Estados Unidos, com amostra de 2824 indivíduos. O outro estudo investigou a relação entre diagnósticos do Diagnostic and Statistical Manual of Mental Disorders (DSM-IV) e 
o ASI, com data de publicação em 2012, com amostra de 252 indivíduos, publicado na Espanha. Ambos os estudos utilizaram a metodologia transversal e também se referiram a usuários de drogas em geral.

Foi realizado um estudo para determinar o potencial do ASI5 como instrumento de triagem para dependência de substâncias. A amostra foi composta por 2.824 participantes. Uma correlação positiva significativa foi encontrada entre escores compostos do ASI e a entrevista diagnóstica de dependência DSM-IV, em ambos os domínios álcool $(r>0.7)$ e drogas $(r>0.5)(p<0.01)$. Os resultados mostraram uma capacidade boa e forte de previsão dos escores composto dos ASI, identificando indivíduos dependentes com aproximadamente $85 \%$ de sensibilidade e $80 \%$ de especificidade(23).

Foi realizado um estudo para avaliar a correlação entre as áreas dos escores de gravidade do entrevistador (EGE) e as áreas dos escores compostos (ECs) do EuropASI. Para avaliar a validade preditiva de ambos os escores, foram entrevistados 252 pacientes. Os resultados indicaram uma alta correlação entre várias áreas do EGE e do ECs, exceto as áreas "legal" e "familiar". Em relação aos resultados preditivos, os pacientes com escore EGE maior que 3 na área "família" foram mais propensos a abandonar o programa em comparação com os pacientes com escore menor que 3 . Os pacientes com ECs superior a 0,34 na área "álcool" tiveram mais probabilidade de abandonar o tratamento(24).

\section{Discussão}

Essa revisão sistemática para avaliar as propriedades psicométricas dos estudos de validação do ASI desde o último estudo realizado em 2004(9) indicou que, embora o ASI tenha sido alvo de críticas, ele continua sendo adaptado e validado em vários países e em diferentes culturas. Neste intervalo, uma nova versão, o ASI6, foi proposta e os escores foram revistos, no intuito de melhorar a sua utilização especialmente para pesquisa. A maioria dos estudos utilizou métodos clássicos de avaliação das propriedades psicométricas do ASI5 e seus derivados, como validade de critério, consistência interna e correlação. No entanto, os escores da sexta versão, os índices resumo das nove áreas, foram desenvolvidos a partir da teoria de resposta ao item não paramétrica(10).

Embora outros instrumentos tenham sido desenvolvidos nos últimos anos, o interesse pelo ASI permanece, pois é considerado um instrumento de avaliação multidimensional que permite o planejamento do tratamento individualizado para o uso problemático de substâncias psicoativas (SPAs). O processo de adaptação de um instrumento utilizado em vários países permite que o pesquisador compare dados obtidos em diferentes amostras, de diferentes contextos, possibilitando uma maior equidade na avaliação, uma vez que se trata de uma mesma medida, a qual avalia o construto a partir de uma mesma perspectiva teórica e metodológica ${ }^{(25)}$. Além disso, tal processo é mais simples e rápido que o da construção de um instrumento, pois envolve menos etapas(26), embora também exija um elevado rigor metodológico(27).

Apesar do ASI6 ter sido desenvolvido em 2006, através de uma ampla revisão do ASI com a proposta de minimizar as limitações da última versão, o ASI5 apareceu na maioria dos estudos ${ }^{(5,7,12,14-17,19-23)}$.

Estudos que utilizaram o ASI5 mostraram baixas correlações dos escores de gravidade do entrevistador (EGE) o que provavelmente reflete a interpretação dos dados pelo entrevistador. Espera-se uma maior concordância entre os escores compostos (ECs) pelo fato desses serem baseados em fórmulas matemáticas e não envolverem a interpretação de entrevistadores. Os EGEs são escalas globais de 5 pontos em cada área do ASI, baseadas na impressão subjetiva do entrevistador sobre o funcionamento passado e recente (últimos trinta dias). O grau de treinamento dos entrevistadores reflete na confiabilidade desses. Evidências mostraram que quando o treinamento tinha menor qualidade ou intensidade, os EGEs também eram menos válidos, por isso, o seu uso passou a ser menos recomendado(1,28-29).

A escala de ECs varia de 0 (nenhum problema) a 1 (problema grave) e os escores são aritmeticamente computados e baseados diretamente na soma bruta de um número fixo de itens em cada área funcional do ASI. Apesar dos ECs teoricamente variarem de 0 a 1 , a escala varia entre as áreas problemáticas e, com isso, as contagens de diferentes domínios não podem ser comparadas diretamente. Além disso, a falta de padronização resulta em dificuldades na interpretação dos $\mathrm{ECs}^{(9-10,16)}$.

Em relação às análises de confiabilidade testereteste, houve uma variação do método empregado. Alguns estudos utilizaram o teste-reteste com diferentes entrevistadores, outros usaram o mesmo entrevistador nas duas entrevistas. Os indicadores de confiabilidade teste-reteste na área "família" foram os mais baixos tanto na quinta versão quanto no ASI6 $(5,14,18)$.

Em relação à consistência interna, o presente estudo considerou que o valor do coeficiente de alfa de Cronbach (a) menor que 0,70 é uma medida problemática(9). Na quinta versão, os três ECs (área "emprego", área "legal", área "família/social") têm consistências baixas em pelo menos cinco estudos diferentes ${ }^{(5,7,12,15-16)}$. Esses dados foram semelhantes ao encontrado na revisão sistemática do $\mathrm{ASI}^{(9)}$. As consistências internas regularmente altas foram relatadas para 3 ECs (área "médica", área "álcool" e área "psiquiátrica"). No ASI6 os SS-Rs também apresentaram consistência interna baixa nas áreas "família" e "Legal"(13,18). 
A literatura apresenta diferente tipos de evidências de validade ${ }^{(30)}$ que visam demonstrar que o instrumento mensura adequadamente o construto que se propõe avaliar e, para tanto, utiliza-se de outras ferramentas (outros instrumentos) para chegar a essa conclusão. As evidências de validade de critério, por sua vez, têm como principal foco investigar o impacto dos escores obtidos por meio do instrumento com outras medidas fortemente relacionadas. Em partes da literatura do ASI, quaisquer associações estatisticamente significantes entre as medidas do ASI e a medida externa foram apresentadas como evidência da validade do $\mathrm{ASI}^{(9)}$. $\mathrm{Na}$ presente revisão dos manuscritos de validação, como pôde ser observado, a maioria dos estudos mostra uma correlação moderada entre os escores sumários do ASI e outros instrumentos, não sendo uniformemente alta. Entretanto as áreas "álcool" e "drogas" foram as que mais apresentaram altas correlações com outros instrumentos considerados medidas externas. Isso é importante pelo fato dessas áreas, que são essenciais ao ASI, demonstrarem altamente confiáveis.

É importante que o ASI apresente medidas de sensibilidade e especificidade dos escores sumários, pois, apenas estudos correlacionais não são suficientes para fornecer uma base para decisões clínicas ${ }^{(9)}$. Uma medida sem sensibilidade deixará de identificar indivíduos com problemas e uma medida sem especificidade deixará de identificar indivíduos que não tenham um problema. Para tanto, uma correlação positiva significativa foi encontrada entre os ECs do ASI5 e os escores do DSM-IV para dependência SPAs em ambos os domínios álcool e drogas. Os resultados mostraram uma previsão moderada a forte: os escores do ASI identificaram indivíduos dependentes com sensibilidade de aproximadamente $85 \%$ e especificidade de $80 \%{ }^{(23)}$. O estudo das propriedades psicométricas da versão reduzida do ASI6, o ASI6 Light, demonstrou para as áreas "álcool" e "drogas" um bom equilíbrio entre especificidade e sensibilidade $(0,93$ e 0,88 , respectivamente) ${ }^{(22)}$.

Os criadores do ASI deram muita ênfase à independência das sete áreas problemáticas do instrumento(1). De fato, a correlação entre as áreas do ASI são geralmente baixas. Portanto, existe exceções, as medidas das áreas "família/social" e "psiquiátrica"(5,10,14,16) e da área "psiquiátrica" e "médica"( $(5,10,13,21)$ às vezes são moderadamente correlacionadas. É importante ressaltar que se quaisquer duas variáveis, por exemplo, uso de drogas e atividades criminosas estão positivamente relacionadas, evidentemente depende de circunstâncias históricas. Em muitas situações é uma característica desejável que as variáveis em uma bateria de teste sejam mutuamente independentes, mas isso geralmente não é um requisito importante, e as correlações entre subescalas não são sinais de validade inadequada ${ }^{(9)}$.

\section{Conclusão}

O presente estudo mostrou um panorama geral das propriedades psicométricas da quinta e sexta versão do ASI desde a última revisão sistemática realizada de maneira bastante crítica. O ASI continua sendo alvo de estudo de validade e confiabilidade em diferentes contextos, cultura e países, variando quanto às formas de aplicação. As semelhanças em suas características psicométricas em diferentes contextos socioculturais permitem o desenvolvimento de pesquisas multicêntricas com um instrumento de aferição confiável e que pode ser aplicado por diferentes profissionais, desde que treinados.

Uma diferença importante entre as duas revisões é a presença da sexta versão nos estudos mais recentes que ocorreram a partir de 2010, que foi elaborada com a finalidade de minimizar os problemas apresentados pelas versões anteriores do ASI, atualizar e expandir suas áreas de avaliação. Da mesma forma, apesar das limitações, o ASI é utilizado amplamente em todo o mundo, por profissionais de diferentes áreas, psicólogos, enfermeiros, assistentes sociais e psiquiatras. Tem auxiliado clínicos e pesquisadores, contribuindo para a elaboração de um plano terapêutico, determinando as prioridades clínicas, bem como o manejo dessas.

\section{Referências}

1. Mc Lellan AL, Luborsky G, Wood GE, O`Brien CP. An improved diagnostic evaluation instrument for substance abuse patients. The Addiction Severity Index. J Nerv Ment Dis. 1980; 168(1): 26-33. http://dx.doi. org/10.1097/00005053-198001000-00006

2. DeJong CA, Willems JC, Schippers GM, Hendriks VM. The Addiction Severity Index: reliability and validity in a Dutch alcoholic population. Int J Addictions. 1995; 30(5): 605-16. http://dx.doi.org/10.3109/10826089509048747 3. Hendriks VM, Kaplan CD, Van Limbeek J, Geerlings, P. The Addiction Severity Index: reliability and validity in Dutch addict population. J Substance Abuse Treat. 1989; 6: 133-41. http://dx.doi.org/10.1016/0740-5472(89)90041-X

4. Krenz S, Dieckmann S, Favrat B, Spagnoli J, Leutwyler J, Schnyder $C$, et al. French version of the addiction severity index (5th Edition): validity and reliability among Swiss opiate-dependent patients. French validation of the Addiction Severity Index. Eur Addiction Res. 2004; 10(4): 173-9. doi: 10.1159/000079839

5. Gerevich J, Bácskai E, Kó J, Rózsa, S. Reliability and Validity of the Hungarian Version of the European Addiction Severity Index: Results of a Multi-Focal Research Project. Psychopathology. 2005; 38(6): 301-9. doi: $10.1159 / 000088918$

6. Senoo E, Ogai Y, Haraguchi A, Kondo A, Ishibashi Y, Umeno $M$, et al. Reliability and validity of the Japanese version of the Addiction Severity Index (ASI-J). Nihon 
Arukoru Yakubutsu Igakkai Zasshi. 2006; 41(4):368-79. https://www.ncbi.nlm.nih.gov/pubmed/17037345

7. Luo W, Wu Z, Wei X. Reliability and validity of the Chinese version of the addiction severity index. J Acquired Immune Deficiency Syndromes. 2010; 53 (1): 121-5. doi: 10.1097/QAI.0b013e3181c7dfca 8. McLellan AT, Kushner H, Metzger D, Peters R, Smith I, Grissom G, et al. A quinta edição do Addiction Severity Index. J Tratamento de Abuso de Substância. 1992; 9 (3): 199-213. https://www.ncbi.nlm.nih.gov/pubmed/1334156 9. Mäkelä K. Studies of the reliability and validity of the Addiction Severity Index. Addiction. 2004; 99(4): 398-410. doi: 10.1111/j.1360-0443.2003.00665.x

10. Cacciola JS, Alterman AI, Habing B, McLellan AT. Recent status scores for version 6 of the Addiction Severity Index (ASI-6). Addiction. 2011; 106 (9): 1588-602. https://doi.org/10.1111/j.1360-0443.2011.03482.x

11. Moher D, Shamseer L, Clarke M, Ghersi D, Liberati $A$, Petticrew $M$, et al. Preferred reporting items for systematic review and meta-analysis protocols (PRISMA-P) 2015 statement. Syst Rev. 2015; 4 (1): 1. doi: $10.1186 / 2046-4053-4-1$.

12. Demirbas H, Ilhan IÖ, Dogan YB, Canatan, A. Reliability and Validity of the Turkish Version of the Addiction Severity index in Male Alcohol dependents. Nöro Psikiyatri Arşivi. 2014; 51(3): 216-21. doi: 10.4274/npa.y6767

13. KesslerF, Cacciola J, Alterman A, FallerS, Formigoni, MLS, Cruz MS, et al. Psychometric properties of the sixth version of the Addiction Severity Index (ASI-6) in Brazil. Revista brasileira de psiquiatria. 2012; 34(1): 24-33. http://dx.doi.org/10.1590/S1516-44462012000100006 14. Sun Z, Chen H, Su Z, Zhou X, Zhang S, Hao W, et al. The Chinese version of the Addiction Severity Index (ASI-C): Reliability, validity, and responsiveness in Chinese patients with alcohol dependence. Alcohol. 2012; 46(8): 777-81. doi: 10.1016/j.alcohol.2012.08.005 15. Haraguchi A, Ogai Y, Senoo E, Saito S, Suzuki Y, Yoshino $A$, et al. Verification of the addiction severity index Japanese version (ASI-J) as a treatmentcustomization, prediction, and comparison tool for alcohol-dependent individuals. Int J Environ Res Public Health. 2009; 6(8): 2205-25. doi: 10.3390/ ijerph6082205

16. Cacciola JS, Alterman AI, McLellan, AT, Lin YT, Lynch KG. Initial evidence for the reliability and validity of a "Lite" version of the Addiction Severity Index. Drug Alcohol Dependence. 2007; 87(2-3): 297-302. doi: 10.1016/j. drugalcdep.2006.09.002

17. Wei LUO, Guo CX, De Lin HAN, Li ZJ. Reliability and validity of Chinese version of the Addiction Severity Index among drug users in the community. Biomedical Environ Sci. 2012; 25(6): 684-9. https://doi. org/10.3967/0895-3988.2012.06.010
18. Díaz MEM, Portilla PG, González MP, Saiz MPA, Bobes BMT, Casares LMJ, et al. Rendimiento psicométrico de la sexta versión del Addiction Severity Index en español (ASI-6). Psicothema. 2010; 22 (3): 513-9. http://www. psicothema. com/psicothema. asp?id $=3761$

19. Reelick NF, Wierdsma AI. The Addiction Severity Index as a predictor of the use of mental health care. Psychol Addictive Behav. 2006; 20(2): 214-8. http:// dx.doi.org/10.1037/0893-164X.20.2.214

20. Alterman AI, Cacciola JS, Habing B, Lynch KG. Addiction severity index recent and lifetime summary indexes based on nonparametric item response theory methods. Psychol Assess. 2007; 19(1): 119-32. doi: 10.1037/1040-3590.19.1.119

21. Denis CM, Cacciola JS, Alterman AI. Addiction Severity Index (ASI) summary scores: Comparison of the recent status scores of the ASI- 6 and the composite scores of the ASI-5. J Substance Abuse Treat. 2013; 45(5): 444-50. doi: 10.1016/j.jsat.2013.06.003 22. Fernandes LR, Colugnati FAB, Sartes LMA. Desenvolvimento e avaliação das propriedades psicométricas da versão brasileira do Addiction Severity Index 6 (ASI-6) Light. J Bras Psiquiatria. 2015; 64(2): 132-9. https://dx.doi.org/10.1590/0047-2085000000068

23. Rikoon SH, Cacciola JS, Carise D, Alterman AI, McLellan, AT. Predicting DSM-IV dependence diagnoses from Addiction Severity Index composite scores. J Substance Abuse Treat. 2006; 31(1): 17-24. doi: 10.1016/j.jsat.2006.03.003

24. López-Goñi JJ, Fernández-Montalvo J, Arteaga A. Predictive validity of the EuropAsi: Clinical diagnosis or composite scoring? J Substance Abuse Treat. 2012; 42: 392-9. http://dx.doi.org/10.1016/j.jsat.2011.09.011

25. Borsa JC, Seize MM. Manual de desenvolvimento de instrumentos psicológicos. São Paulo: Vetor; 2017.

26. Pacico JC. Como é feito um teste? Produção de itens. In: Hutz CS, Bandeira DR, Trentini CM, editores. Psicometria. Porto Alegre: Artmed; 2015. p. 55-70

27. Borsa JC, Damásio BF, Bandeira DR. Adaptação e validação de instrumentos psicológicos entre culturas: algumas considerações. Paideia. 2012; 22 (53): 423-32. http://dx.doi.org/10.1590/S0103-863X2012000300014 28. Kessler, F, Pechansky, F. Manual de Aplicação da Sexta Versão da Escala de Gravidade de Dependência [Manual]. Brasília; Secretaria Nacional de Politicas Sobre Drogas (SENAD), 2006. Disponível em: http://www. obid.senad.gov.br/portais/OBID/biblioteca/documentos/ Informacoes_drogas/escalas/327585.pdf

29. Alterman AI, Bovasso GB, Cacciola JS, Mc Dermott PA. A comparison of the predictive validity of four sets of baseline ASI summary indices. Psychol Addict Behav. 2001; 15: 159-62.

30. American Education Research Association [AERA], American Psychological Association [APA], National 
Council on Measurement in Education [NCME]. Standards for educational and psychological testing. Washington, DC: American Psychological Association; 2014.

31. Sordi AO, Sartes LAM, Kessler FHP. Escala de gravidade de dependência. In: Gorenstein C, Wang YP, Hungerbühler I., editors. Instrumentos de avaliação em saúde mental. Porto Alegre: Artmed; 2016. p. 218-24.

\section{Contribuição dos autores}

Concepção e planejamento do estudo: Lídia Reis Fernandes, Laisa Marcorela Andreoli Sartes. Obtenção dos dados: Lídia Reis Fernandes, Laisa Marcorela Andreoli Sartes, Maira Leon Ferreira. Análise e interpretação dos dados: Lídia Reis Fernandes, Laisa Marcorela Andreoli Sartes, Maira Leon Ferreira. Redação do manuscrito: Lídia Reis Fernandes, Laisa Marcorela Andreoli Sartes, Maira Leon Ferreira. Revisão crítica do manuscrito: Lídia Reis Fernandes, Laisa Marcorela Andreoli Sartes, Maira Leon Ferreira.

Todos os autores aprovaram a versão final do texto.

Conflito de interesse: os autores declararam que não há conflito de interesse. 